GA-A22720

CONF-971065--

\title{
THE DIII-D ECH MULTIPLE GYROTRON CONTROL SYSTEM
}

by
D. PONCE, J. LOHR, J.F. TOOKER, W.P. CARY, and T.E. HARRIS

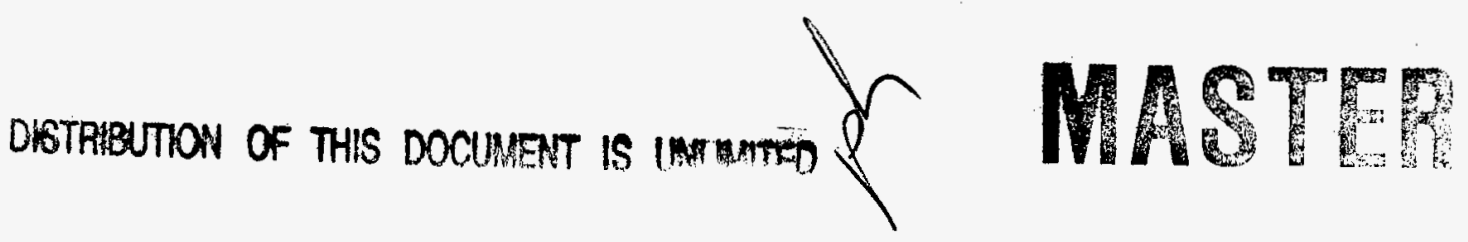

19980401055

RECEIVED

FFR 101998

OSTI

NOVEMBER 1997 


\section{DISCLAIMER}

This report was prepared as an account of work sponsored by an agency of the United States Government. Neither the United States Government nor any agency thereof, nor any of their employees, makes any warranty, express or implied, or assumes any legal liability or responsibility for the accuracy, completeness, or usefulness of any information, apparatus, produce, or process disclosed, or represents that its use would not infringe privately owned rights. Reference herein to any specific commercial product, process, or service by trade name, trademark, manufacturer, or otherwise, does not necessarily constitute or imply its endorsement, recommendation, or favoring by the United States Government or any agency thereof. The views and opinions of authors expressed herein do not necessarily state or reflect those of the United States Government or any agency thereof. 
GA-A22720

\title{
THE DIII-D ECH MULTIPLE GYROTRON CONTROL SYSTEM
}

\author{
by \\ D. PONCE, J. LOHR, J.F. TOOKER, W.P. CARY, and T.E. HARRIS
}

This is a preprint of a paper to be presented at the 17th IEEE/NPSS Symposium on Fusion Engineering, October 6-11, 1997, San Diego, California and to be published in the Proceedings.

\author{
Work supported by \\ the U.S. Department of Energy \\ under Contract No. DE-AC03-89ER51114
}

GA PROJECT 3466

NOVEMBER 1997 


\title{
The DIII-D ECH Multiple Gyrotron Control System*
}

\author{
Dan Ponce, John Lohr, J.F. Tooker, W.P. Cary and T.E. Harris \\ General Atomics \\ P.O. Box 85608, San Diego, California 92186-9784
}

\begin{abstract}
DIII-D's ECH upgrade with $1 \mathrm{MW}, 110 \mathrm{GHz}$ gyrotrons is ongoing, and with it, an upgrade of the control system. The ECH Multiple Gyrotron Control System uses software distributed among networked computers, interfaced to a programmable logic controller (PLC), the timing and pulse system, power supplies, vacuum and wave guide controls, and instrumentation. During DIII-D operations, the system will allow a chief and a co-operator to control and monitor a number of gyrotrons from different manufacturers.
\end{abstract}

The software, written using LabVIEW, allows for remote and multiple operator control. Thus any supported computer can become a control station and multiple projects can be simultaneously accommodated. Each operator can be given access to the controls of all gyrotrons or to a subset of controls. Status information is also remotely available.

The use of a PLC simplifies the hardware and software design. It reduces interlock and control circuitry, includes monitoring for slow analog signals, and allows one software driver to efficiently interface to a number of systems. In addition, the interlock logic can be easily changed and control points can be forced as needed.

The pulse system is designed around arbitrary function generators. Various modulation schemes can be accommodated, including real-time control of the modulation.

This discussion will include the hardware and software design of the control system and its current implementation.

\section{SYSTEM OVERVIEW}

The ECH gyrotron installation at DIII-D currently consists of a Gycom Centaur GLGF 110/1.0 and a CPI (formerly Varian) VGT-8011A. A second VGT-8011A is expected in 1998. The hardware installed to host the control and monitoring system software for these tubes consists of a Sun SPARC workstation, three PC-compatible computers, three NCD HMX X-terminals, and a Siemens Simatic TI545 PLC.

During DIII-D operations, two ECH operators are expected to control all on-line gyrotrons. The ECH chief operator will be responsible for interfacing with the
DIII-D control room and setting operating parameters, including pulse timing. The co-operator will monitor gyrotron and system performance, ensuring that commands are executed properly. During commissioning and special projects, each gyrotron might have its own operator.

The operator's control panel connects to the system over the computer network. The operator can reside where it is most convenient, including the DIII-D or ECH control rooms. The control panel allows the operator to set operating parameters for any combination of gyrotrons. Several instances of the control panel can exist simultaneously to accommodate the number of operators. A central control server sets operator privileges to prevent conflicts. The gyrotron status and waveforms are also available remotely.

\section{CONTROL SYSTEM SOFTWARE}

The control system software provides the operator interface for the control and monitoring of all gyrotrons. At the time the task of designing the system was initiated, only one gyrotron was in use, however, the system is specified to accommodate as many as twelve gyrotrons.

A major factor in determining the design of the control software was the recognition that this software will need to accommodate the hardware changes that will occur as the number of installed gyrotrons increases. These changes would include the number, and maybe even the type of host computers. It was decided to take advantage of General Atomics' extensive computer network to design the control software around limited task modules interconnected via the internet. This will provide for flexibility as the system grows. This flexibility is enhanced by the use of the multi-platform LabVIEW programming environment [1]. Software written using LabVIEW can be rather easily transported between Windows, Mac, SunSolaris, and HP-UX operating systems, if it is written with this goal in mind.

The overall layout of the control software is show in Fig. 1. Data generally flows in one direction, initiating and ending with the operator. The state of the system is determined by the control server and its command buffers,

\footnotetext{
*Work supported by U.S. Department of Energy under Contract No. DE-AC03-89ER51114.
} 


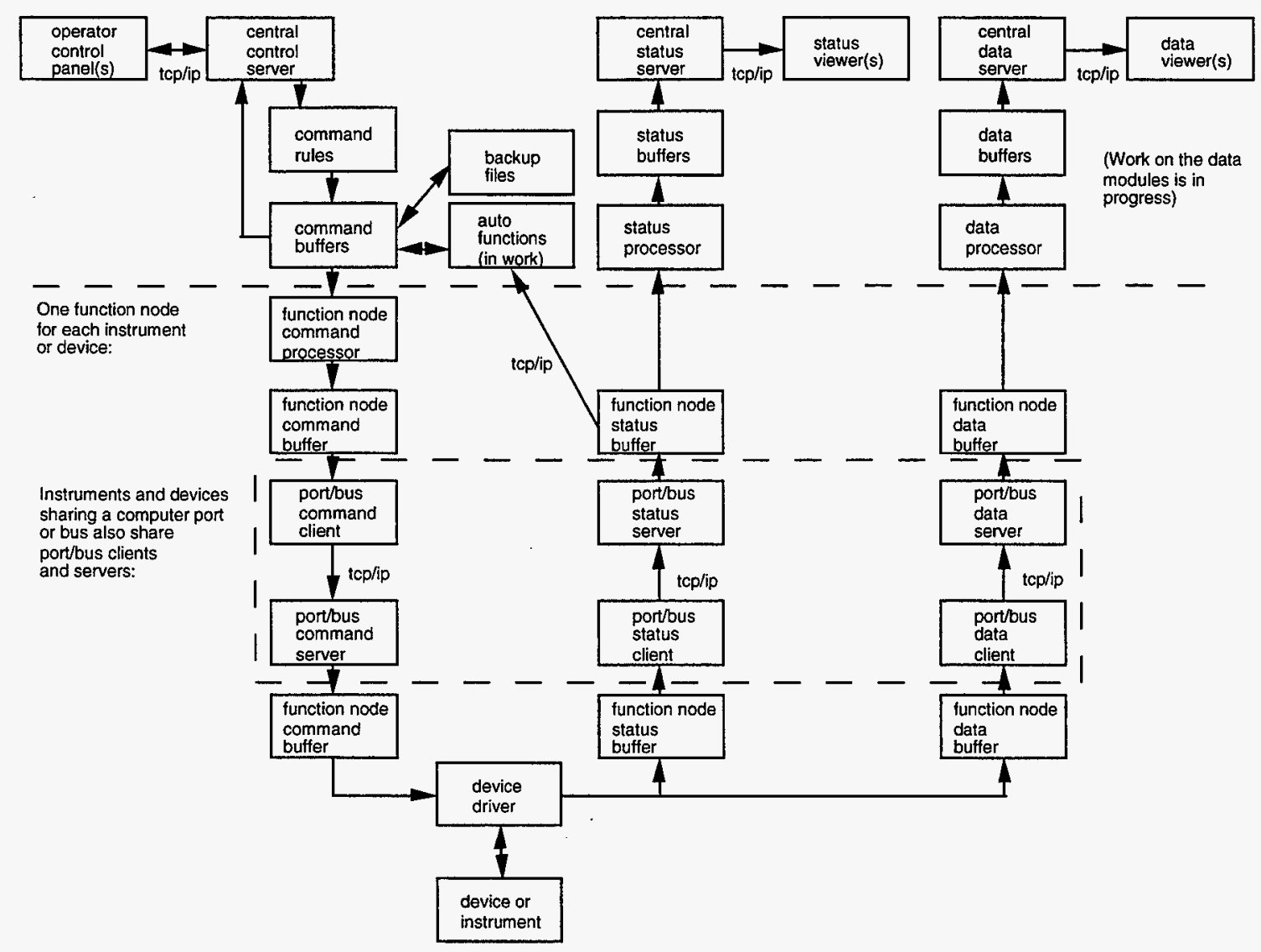

Fig. 1. Control system software layout.

with input from the operator. Hardware control is handled by the function nodes and there is one function node for each hardware unit. The function nodes act upon the commands from the control server. Status and data flows from the function nodes to the status and data servers, respectively. These servers pass the information on to viewing stations, where the operator or other interested parties can monitor the response of the system.

It should be noted that safe system operation does not depend upon the operator monitoring the response of the system. The control server will only issue operating parameters that its built-in rules determine are safe. Ultimately the hardware based fault and interlock systems prevent unsafe operation. The operator's monitoring role is to verify that commands were successfully passed on to the hardware, without activating the fault and interlock systems, and that the software is responding normally.

There are several aspects of the software design that enhance robustness. Examples include the use of isolated software modules with limited tasks, the self monitoring TCP/IP interfaces, and the management of computer I/O access.

An example of a limited task software module is the operator's control panel. The primary task of this module is to send the operator's commands to the command server. It performs two other related tasks. It echoes back the operator's commands, as accepted by the command server, and allows the operator to monitor the commands from other logged-in operators. The limited task nature of the module means that its size will be minimized, reducing memory problems. The code can also be more focused, enhancing maintainability.

This module is also isolated from other modules, meaning that it has its own memory space. It communicates with other modules using TCP/IP protocol. Being isolated means that the module can take advantage of the operating system's memory protection and task scheduling functions. On an operating system with robust memory protection, the module will be isolated from memory problems caused by other processes. On an operating system with strict time sliced task scheduling, the module can run more evenly than if it were included in a more complex module.

The TCP/IP links throughout the control system software are self monitoring. This is implemented by checking the format of the received data and errors are indicated by returning an error code or, in most cases, breaking the TCP/IP link. Modules which break the link are self resetting. They will attempt to reestablish the link until successful. Each link is assigned a set of network ports 
which the modules will scan looking for working ports. In practice, these links are very robust.

Access to computer $\mathrm{I} / \mathrm{O}$ is strictly managed by the control system software, not allowing such management to be deferred to the task management of LabVIEW nor that of the operating system. This is implemented by placing all routines which access the same $\mathrm{I} / \mathrm{O}$ bus or port inside a scheduling routine. Each $\mathrm{I} / \mathrm{O}$ routine is written following a similar template, as follows. The routine first checks a memory buffer to see if new commands have been received. If so, it acts upon the command set and then collects reportable status and data. Finally, the routine buffers the status and data. The scheduling routine then calls the next I/O routine for execution. In parallel, TCP/IP routines receive new commands into the memory buffer and send changed status and data. This arrangement yields an additional benefit. $\mathrm{W} / \mathrm{O}$ routines using the same bus or port will share memory space, therefore, using the memory buffer structure, I/O routines can interact at a low level. However, since the I/O routines do not execute simultaneously, they can still be swapped to minimize memory usage.

\section{PROGRAMMABLE LOGIC CONTROLLER (PLC)}

A Siemens Simatic TI545 PLC is used for interlock logic, slow signal monitoring, and process control. Alternately, these functions could have been implemented using hardware or a desktop computer. The programmable logic of the PLC allows for faster changes to the interlock or process control logic through software than could be achieved with just hardware. It unburdens the desktop computer for more appropriate tasks, and is in fact more reliable for interlock monitoring and process control. With the number of I/O channels in the ECH system, the PLC is heavily favored. Nearly 400 channels have been assigned for three gyrotrons. Fig. 2 illustrates some of the systems that interface with the PLC.

\section{PULSE SYSTEM}

The timing signals required during a gyrotron pulse are generated by multiple channel digital delay pulse generators. The Gycom system uses a Stanford Research Systems DG535. This generator provides four transition outputs and two pulse outputs. The Berkeley Nucleonics $500 \mathrm{E}$, with eight pulse outputs, is being evaluated for use with the CPI system. The generators provide where applicable, the reset pulse, digitizer triggers, heater boost signal, cathode high voltage pulse, and gun anode pulse. A master timing signal which is received from the DIII-D control room, triggers these generators during DIII-D experiments.

The cathode and gun anode pulses are sent to pulse control modules which also receive input from the fault and interlock hardware. The pulse control modules then decide whether to forward the cathode and gun anode pulses, to interrupt or terminate the pulses, or to send a crowbar signal to the high voltage power supply.

The reference waveforms used to control rf generation are provided by Wavetek 395 arbitrary waveform generators.

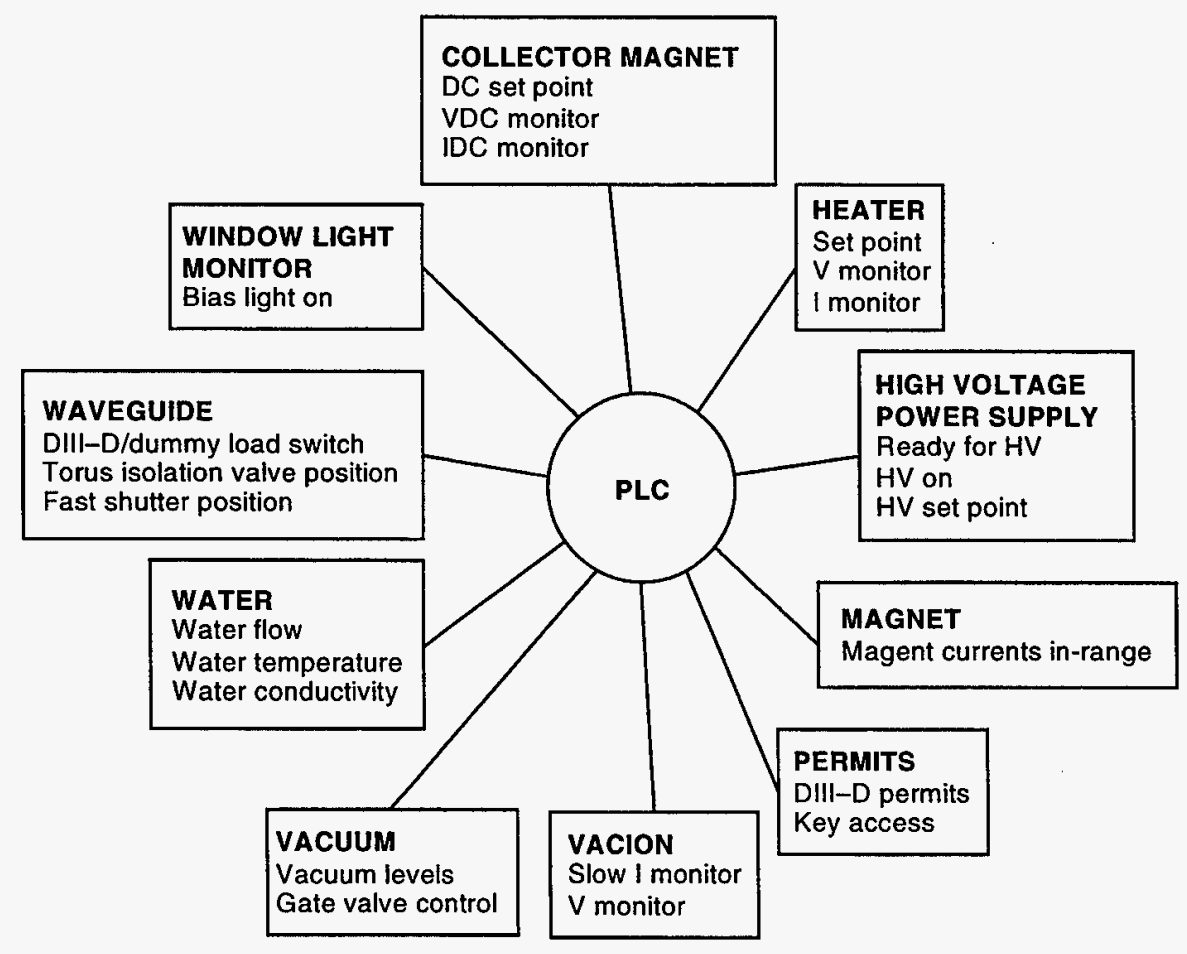

Fig. 2. Systems interfaced with the PLC. 
In the Gycom system, the reference waveform is used to regulate cathode voltage. In the CPI system, it is used to regulate gun anode voltage. The use of the Wavetek 395 allows for several modes of operation. The system is currently programmed to provide a standard square pulse with an initial ramp if desired. Modulated pulses are also available with square, sawtooth, and sinusoidal waveforms. The high and low amplitude and the location of the slope change in the waveform can be specified. Fig. 3 shows a sinusoidal rf pulse from the Gycom gyrotron.

More sophisticated modulation schemes are possible with the Wavetek 395. An AM input is available and so the reference waveform can be programmed to follow an external signal. It is also possible to trigger advancement through a preprogrammed sequence. Thus, the reference waveform can be altered in response to an external trigger. This versatility in modulation schemes is one of the reasons the Wavetek 395 was selected to generate the reference waveform.

\section{REFERENCES}

[1] A.L. Wright, J. Tooker, J.C. Allen, W.P. Cary, and T.E. Harris, "ECH control system for new 1MW 110 GHz gyrotrons at DIII-D," Proc. 15th IEEE/NPSS Symp. on Fusion Engineering, Vol. 2, p. 889, 1995.

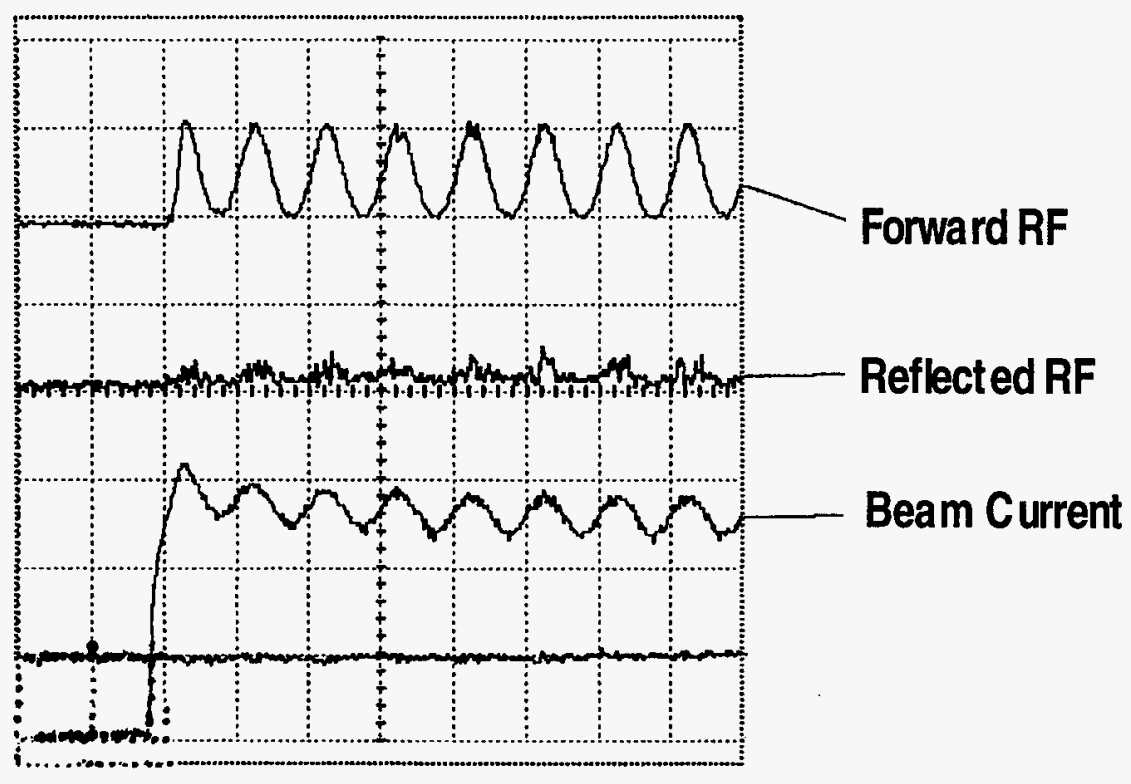

Fig. 3. $100 \mathrm{~Hz}$ sinusoidal if power output from the Gycom gyrotron. 
M98002643

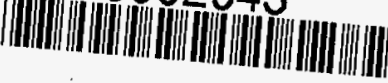

Report Number (14) $\frac{G A-A 22720}{\text { CONE-971065 }}$

Jubl. Date (11) 199711

sponsor Code (18) $\frac{D O E}{4 C-400, D O E / E R}$

DOE 